\title{
Elastic large muscular vessel wall engineered with bone marrow-derived cells under pulsatile stimulation in a bioreactor
}

\author{
ZHI CHENG XU ${ }^{1}$, QUN ZHANG ${ }^{1}$ and $\mathrm{HONG} \mathrm{LI}^{2}$ \\ ${ }^{1}$ Department of Plastic and Reconstructive Surgery, Shanghai 9th People's Hospital, School of Medicine, \\ Shanghai Jiaotong University, Shanghai 200011; ${ }^{2}$ Department of Life Information and Instrument Engineering, \\ Hangzhou Electronic Science and Technology University, Hangzhou, Zhejiang 310058, P.R. China
}

Received November 2, 2014; Accepted July 3, 2015

DOI: $10.3892 / \mathrm{mmr} .2015 .4147$

\begin{abstract}
Bone marrow-derived cells (BMCs) have demonstrated their ability to differentiate into multiple cell lineages and may be a promising cell source for vascular tissue engineering. Although much progress has been made in the engineering of small blood vessels ( $<6 \mathrm{~mm}$ in diameter) with biodegradable materials such as polyglycolic acid (PGA), it remains a challenge to engineer large vessels $(>6 \mathrm{~mm}$ in diameter) due to unsatisfactory biomechanical properties. The present study was to engineered an elastic large vessel wall (6 $\mathrm{mm}$ in diameter) using a PGA unwoven fibre scaffold covered with BMCs from canine humeri. The cell-PGA sheet was then loaded into a bioreactor designed for the present study, with dynamic pulsatile culture conditions to mimic the physiological vessel environment. After four weeks of the pulsatile stimuli culture, an elastic vessel wall was formed. Histological analyses demonstrated that layers of smooth muscle-like cells and well-oriented collagenous fibres were evenly oriented in the dynamic group. By contrast, disorganised cells and randomly collagenous fibres were apparent in the static group. Furthermore, the engineered vessel wall in the dynamic group exhibited significantly improved biomechanical properties compared with those in static culture group. The approach developed in the present study was demonstrated to have promising potential to be used for the engineering of large vessel as well as other smooth muscle cell-containing tissues, including bladder, urethral and intestinal tissues.
\end{abstract}

Correspondence to: Professor Qun Zhang, Department of Plastic and Reconstructive Surgery, Shanghai 9th People's Hospital, School of Medicine, Shanghai Jiaotong University, 639 Zhi Zao Ju Road, Shanghai 200011, P.R. China

E-mail: zhangqunmd@163.com

Key words: vascular tissue engineering, bone marrow-derived cells, bioreactor

\section{Introduction}

Numerous types of congenital heart defect and peripheral vascular disease require repairing with vascular conduits. Autologous vascular grafts often cannot be used for this purpose due to limited sources, donor site morbidity or even damage from a pre-existing disease (1). However, synthetic materials, including expanded polytetrafluoroethylene (ePTFE) and polyethylene terephthalate fibre (Dacron) have been widely used in cases of large vessel lesions (2). However, these materials are usually non-degradable and lack the potential for growth. Furthermore, these grafts often suffer from immunologic and thrombotic complications, including rejection, stenosis, thrombosis, calcium deposition and infection, as revealed by long-term follow-up studies (3).

Tissue engineering, in which regenerating vascular grafts are constructed using cells and degradable scaffolds, allows for an alternative approach to resolve these problems (4). Promising results have been reported using tissue-engineered blood vessels (TEBVs) (5), but the repairable vascular defects are limited to small diameters $(<6 \mathrm{~mm})$ under low blood pressure (6-8). In cases with larger vessels ( $>6 \mathrm{~mm}$ in diameter), the biomechanical properties of these engineered grafts cannot sufficiently withstand the powerful impact of blood flow (9).

In addition, tissue engineering approaches are limited by the availability of autologous cells. These cells have often shown a limited replicative capacity, particularly when they originated from older donors. Therefore, it is necessary to find an autologous source of progenitor vascular cells with a high proliferative capacity for the preparation of TEBVs. A possible solution is the use of bone marrow-derived cells (BMCs), which have demonstrated multiple cell differentiation abilities and a high proliferation potential. In previous studies, BMCs have been found to differentiate into smooth muscle (SM)-like cells or endothelial cells in vitro (10-15). Furthermore, when compared with embryonic stem cells, BMCs are not confounded by ethical considerations and are readily available for research. In addition, bone marrow aspiration is less invasive and carries a reduced risk of morbidity at the donor site and may thus be a promising alternative cell source for vascular tissue engineering (16-18). 
The aim of the present study was to engineer a large vessel (6 mm in diameter) with canine BMCs and a polyglycolic acid (PGA) scaffold in a pulsatile flow bioreactor for four weeks of dynamic cultivation. PGA is a useful scaffold material, as its rapid degradation prevents the accumulation of degraded fragments in the body (4). The mechanical properties of the vessel engineered in the present study were then assessed in order to test the applicability of the developed method to the engineering of various organ parts, including blood vessels and large muscular tubular structures, such as the oesophagus, intestine or ureter.

\section{Materials and methods}

Experimental animals. Twelve adult beagles (6 months old) were purchased from Chenhang Experimental Animal Raising Farm (Shanghai, China). The experimental protocol was approved by the Animal Care and Experimental Committee of Shanghai Jiao Tong University School of Medicine (Shanghai, China). All the protocols of animal handling were approved by the Research Ethical Committee of the hospital.

BMCs isolation and culture. Following sacrifice via intravenous administration of $50 \mathrm{mg} / \mathrm{kg} 1 \%$ alpha-chloralose (LongDan Pharmaceutical and Chemical Co., Shanghai, China), bone marrow (10 $\mathrm{ml}$ from each dog) was aspirated from the humeri of the dogs and mixed with heparin (100 unit heparin/ml bone marrow; Sigma-Aldrich, St. Louis, MO, USA). Four healthy canine abdominal arteries were donated for use in the study by the Cardiovascular Research Centre of the Shanghai 9th People's Hospital (Shanghai, China). The bone marrow and heparin mixture was centrifuged on a Ficoll-Paque density gradient (Amersham Bioscience, Arlington Heights, IL, USA) for $20 \mathrm{~min}$ at $352 \mathrm{x} \mathrm{g}$. Bone marrow mononuclear cells (BMMNCs) were isolated from the buffy coat layer between the Ficoll-Paque reagent and blood plasma component and washed three times in phosphate-buffered saline (PBS; Sigma-Aldrich) solution. The BMMNCs were cultured in low-glucose Dulbecco's modified Eagle's medium (LG-DMEM; Gibco-BRL, Invitrogen Life Technologies, Inc., Carlsbad, CA, USA) supplemented with $10 \%$ fetal bovine serum (FBS; HyClone Laboratories, Logan, UT, USA), $100 \mathrm{U} / \mathrm{ml}$ penicillin (Sigma-Aldrich), and $100 \mathrm{mg} / \mathrm{ml}$ streptomycin (Sigma-Aldrich) at $37^{\circ} \mathrm{C}$ with $95 \%$ humidity and $5 \% \mathrm{CO}_{2}$. The medium was replaced twice a week. The characterization of the BMCs was determined by their $\mathrm{CD}$ marker profiles and their ability to differentiate into osteogenic, adipogenic and chondrogenic lineages as previously reported (data not shown) (19). BMCs at passage 2 were used for the vessel construction. Prior to seeding, the identity of the BMCs was confirmed by immunofluorescent staining with mouse monoclonal anti- $\alpha$-smooth muscle actin ( $\alpha$-SMA; cat. no. C6198; Sigma-Aldrich), rabbit monoclonal anti- $\alpha$-calponin (cat. no. ab46794; Abcam, Cambridge, UK) and mouse monoclonal anti-smooth muscle myosin heavy chain (SM-MHC; cat. no. M7786; Sigma-Aldrich), all at a dilution of 1:100.

Culture of BMC-PGA sheets in dishes. A total of $30 \mathrm{mg}$ unwoven PGA fibres (Albany International Research Company Inc.,
Albany, NY, USA) were constructed into a 35x30x2 mm mesh. The scaffold was first soaked in $75 \%$ ethanol (Sigma-Aldrich) for $2 \mathrm{~h}$. Subsequently, it was washed three times with PBS and incubated in DMEM for $10 \mathrm{~min}$. The medium was removed, and the scaffold was incubated at $37^{\circ} \mathrm{C}$ prior to being used. The $\mathrm{BMCs}$ were collected and re-suspended in the culture medium at a density of $6 \times 10^{7}$ cells $/ \mathrm{ml}$. Finally, $3 \times 10^{7}$ cells were evenly seeded onto the PGA mesh in 100-mm culture dishes (Falcon, Oxnard, CA, USA). To accomplish the complete adhesion of the BMCs to the fibres, the cell-scaffold constructs were then incubated at $37^{\circ} \mathrm{C}$ with $95 \%$ humidity and $5 \% \mathrm{CO}_{2}$ for $4 \mathrm{~h}$. Thereafter, sufficient DMEM with $10 \%$ FBS was added to the dish to cover the construct. The cell-PGA sheet was incubated for another five days prior to being placed into the bioreactor.

Scanning electron microscopic (SEM) observation. For the observation of the growth conditions of the cells on the PGA, parts of the cell-PGA sheets were harvested for an SEM examination after five days of culture. The samples were first pre-fixed with $2.5 \%$ glutaraldehyde (Sigma-Aldrich) for $2 \mathrm{~h}$ at $4^{\circ} \mathrm{C}$ and then washed three times with PBS. Thereafter, the samples were post-fixed with $1 \%$ osmic acid (Sigma-Aldrich) for $2 \mathrm{~h}$ at $4^{\circ} \mathrm{C}$. After washing with PBS three more times, the samples were dehydrated through an ethanol gradient (Sigma-Aldrich), mounted, sputter-coated with gold and then viewed under a scanning electron microscope (Quanta 200; FEI, Hillsboro, OR, USA).

Vessel reactor preparation. The blood vessel reactor (Hongli, Shanghai, China) was a closed-loop perfusion system that primarily consisted of a culture chamber, silicone tubes, a media reservoir, a peristaltic pump, a magnetic valve and a pressure meter. The culture chamber including the four culture dishes was composed of transparent polycarbonate with four lids for gas exchange (Fig. 1). The 6-mm outer diameter medical-grade silicone tube was threaded through the side arms of the chamber and secured. Sterile PBS was pumped from the media reservoir through the silicone tubes and back into the reservoir. The circulation was managed by a peristaltic pump and a magnetic valve. The former provided continuous fluid flow at a rate of $0.01-1,000 \mathrm{ml} / \mathrm{min}$. The latter was periodically closed under the control of a custom-made computer. The periodic closing of the valve produced a pulsed flow in the bioreactor that attempted to mimic the circulation system in vivo (blood vessel bioreactor: Designed and manufactured by Professor Hong Li; Department of Life Information and Instrument Engineering, Hangzhou Electronic Science and Technology University, Hangzhou, Zhejiang, China). Therefore, it was possible to adjust the corresponding radial distension rate of the silicone tube by modifying the flow rate. The pressure meter showed the changes in the media pressure, which was necessary for the modification of the circulation system. Prior to use, the culture chamber was sterilised with ethylene oxide.

Dynamic culture in the vessel reactor. After being cultured in dishes for five days, the cell-PGA sheets were wrapped around the silicone tubes and further fixed by biodegradable sutures (Ethicon, Inc., Somerville, NJ, USA; Fig. 2B) in the culture chamber of the vessel reactor. The sheets were covered with DMEM containing $10 \%$ FBS in the chamber. Thereafter, the 


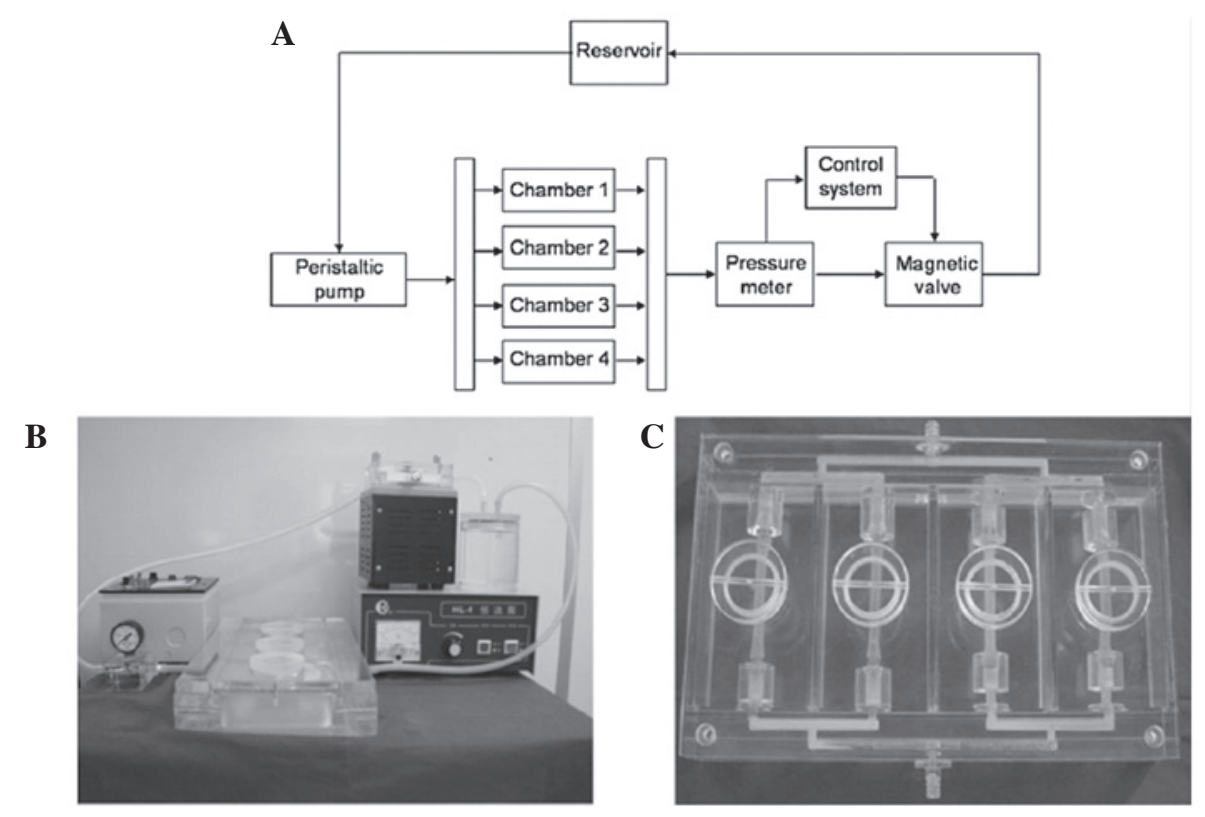

Figure 1. Bioreactor used in the present study. (A) Schematic representation of the closed-loop perfusion system. (B) Fully assembled system consisting of a culture chamber, a media reservoir, a peristaltic pump, a magnetic valve that is controlled by a custom-made computer and connecting silicone tubes. (C) Image of the culture chamber made of transparent polycarbonate with a lid for gas exchange. A medical-grade silicone tube with an outer diameter of 6 mm was threaded through the side arms of the chamber and secured.

culture chamber was connected to the entire vessel reactor. Sterile PBS flowed through the silicone tubes in a pulsatile manner at a frequency of 75 beats $/ \mathrm{min}$. The flow rate was gradually increased and adjusted $(70-80 \mathrm{ml} / \mathrm{min})$ to reach a radial distension of $\sim 5 \%$ of the original diameter of the constructs. The culture chamber was placed in the incubator at $37^{\circ} \mathrm{C}$ for four weeks, and the culture media was changed twice a week. Cell-PGA constructs cultured under static conditions without pulsatile radial stress were used as controls.

Histological analyses. After four weeks of culture, the engineered vessel walls were harvested, fixed in $10 \%$ formalin (Sigma-Aldrich) and embedded in paraffin (Sigma-Aldrich). The vessels were then sequentially cut into $4-\mu \mathrm{m}$ sections. The sections were then subjected to haematoxylin and eosin (HE) or Masson's trichrome and Gomori staining (all stains were from Sigma-Aldrich).

Biomechanical testing and hydroxyproline assay. A tensile tester (4456; Instron, Norwood, MA, USA) was used in the biomechanical tests. As previously reported (20), the engineered vessel wall was placed between the jaws of the tester with a constant elongation $(3 \mathrm{~mm} / \mathrm{min})$ applied along the longitudinal axis until rupture occurred to determine the maximum tensile force. To determine the suture-holding strength, 5-0 polypropylene sutures (Ethicon, Inc.) were placed in four quadrants of the vessel wall at a location of $1 \mathrm{~mm}$ from the vessel edge, followed by constant elongation $(3 \mathrm{~mm} / \mathrm{min})$ along the longitudinal axis of the vessel in the same tester until the sutures pulled through the edge of the vessel (21). For the hydroxyproline assay, the vessel wall was dried and weighed. The total hydroxyproline content of each vessel was determined by a colorimetric assay, as described by Reddy and Ewemeka (22). In the current study, a Sigma-MAK008, Hydroxyproline Assay Kit (Sigma-Aldrich) and Genesys 20
Spectrophotometer (Z376027; Sigma-Aldrich) were used. Native canine abdominal arteries $(6 \mathrm{~mm}$ in diameter) served as the normal controls in all of the above tests.

Statistical analysis. Each experiment was repeated at least three times. As the original data were normally distributed, the results were expressed as the mean \pm standard deviation. Significant differences between values were determined using Student's $t$-test. A P-value less than 0.05 was considered to indicate a statistically significant difference. All of the statistical analyses were performed using SPSS version 16 (SPSS, Inc., Chicago, IL, USA).

\section{Results}

Culture of BMCs. After 4-5 days of culture in the plates, the BMCs were elongated and showed a spindle-like shape. Within another 2-3 days, the cells reached confluency and were subsequently passaged onto a fresh plate. Immunofluorescent staining of the cells at passage 2 showed that nearly half of the cells were positive for anti-alpha-SMA, calponin and SM-MHC staining (Fig. 2).

Culture of BMC-PGA constructs in dishes. Passage-2 BMCs were seeded onto the PGA mesh. After $24 \mathrm{~h}$ of culture, the cells began to spread and extended along the length of the fibres. After another five days of culture in the dishes, a cell-PGA sheet was formed (Fig. 3A). Micrographs showed that the abundant BMCs had adhered to the PGA fibres, with secreted extracellular matrix (ECM) filling the space between the fibres (Fig. 3B), which was further confirmed by SEM observation (Fig. 3C).

Dynamic culture in the vessel reactor. After being placed into the bioreactor, the BMC-PGA constructs were incubated for 
A

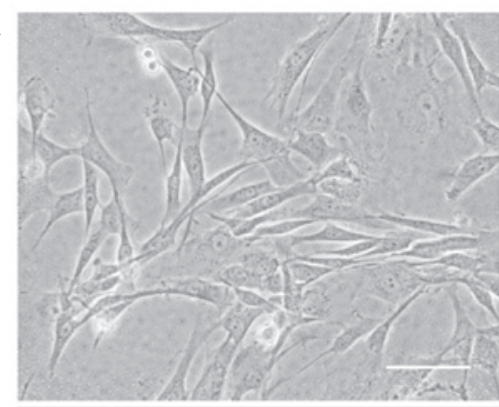

C

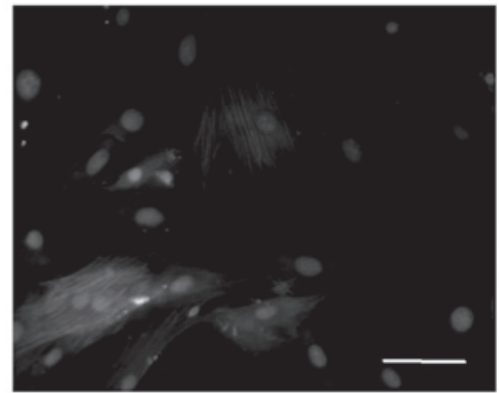

B
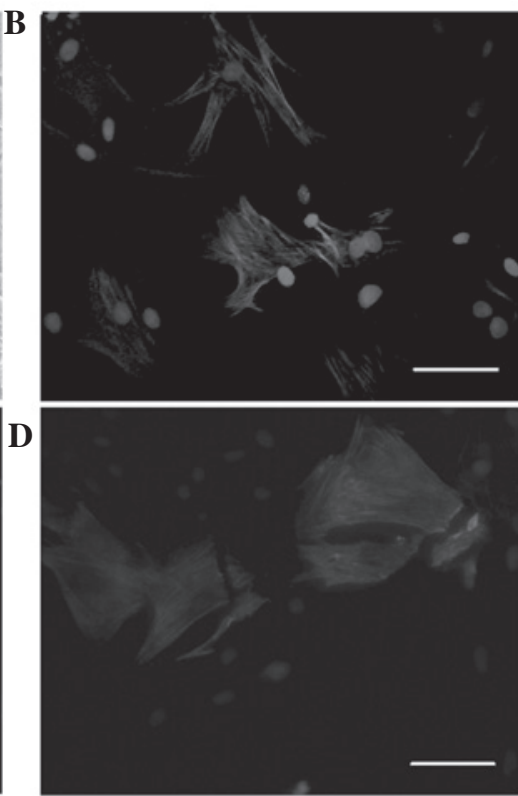

Figure 2. Characterization of bone marrow-derived cells at passage 2. (A) Morphology of the cells under a light microscope. Immunofluorescent staining showed that nearly half of the cells were positive for (B) $\alpha$-smooth muscle actin, (C) calponin and (D) smooth muscle myosin heavy chain. Scale bars, $50 \mu$ m.

A

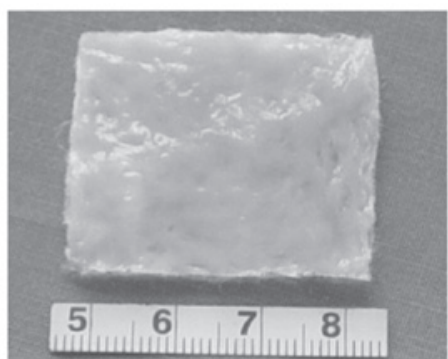

C

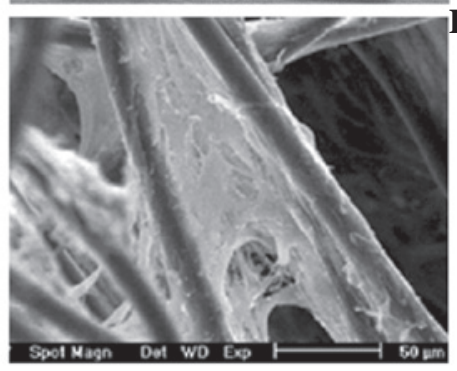

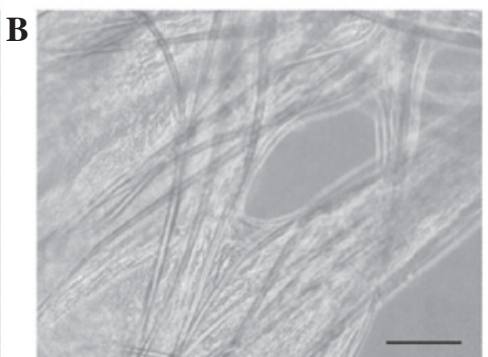

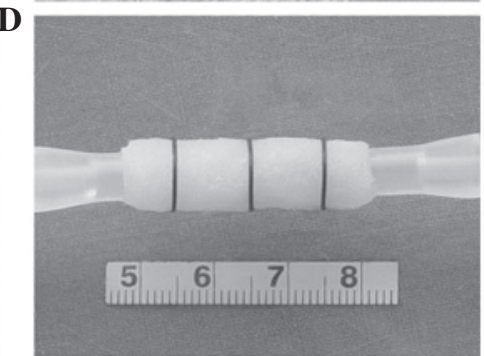

Figure 3. Cell-scaffold constructs cultured in dishes. (A) BMCs were seeded on a PGA unwoven fiber mesh and cultured for five days in a dish. (B) Following five days of culturing, cell - PGA constructs were observed by microscopy (scale bar, $100 \mu \mathrm{m}$ ). Plenty of BMCs adhered to the PGA fibers with secreted ECM filling the space between the fibers. (C) Scanning electron microscopic image of the production of ECM by BMCs on PGA fibers at day five of culturing (scale bar, $50 \mu \mathrm{m}$ ). (D) The cell-PGA sheet was wrapped around a silicone tube in the culture chamber of a vessel reactor and secured with biodegradable sutures. BMC, bone marrow cell; PGA, polyglycolic acid; ECM, extracellular matrix.

A

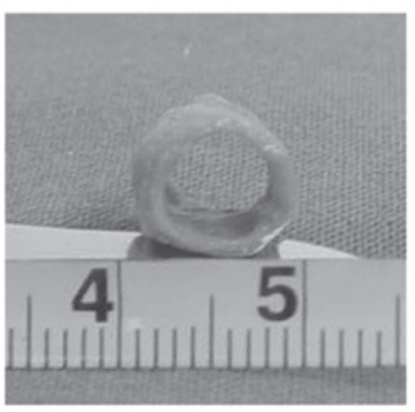

B

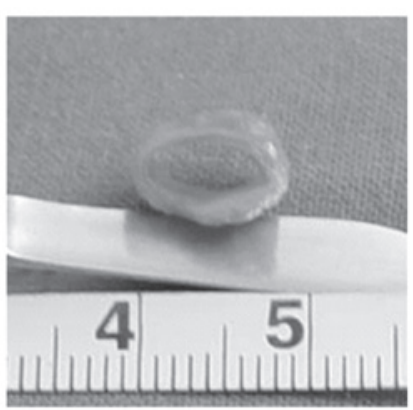

Figure 4. Gross view of the engineered vessels. (A) Vessel walls with a round lumen (6 mm in diameter) were formed after four weeks of culture with pulsatile stimulation. (B) Vessel walls with a collapsed lumen and rough surface are observed after culture without pulsatile stimulation. 

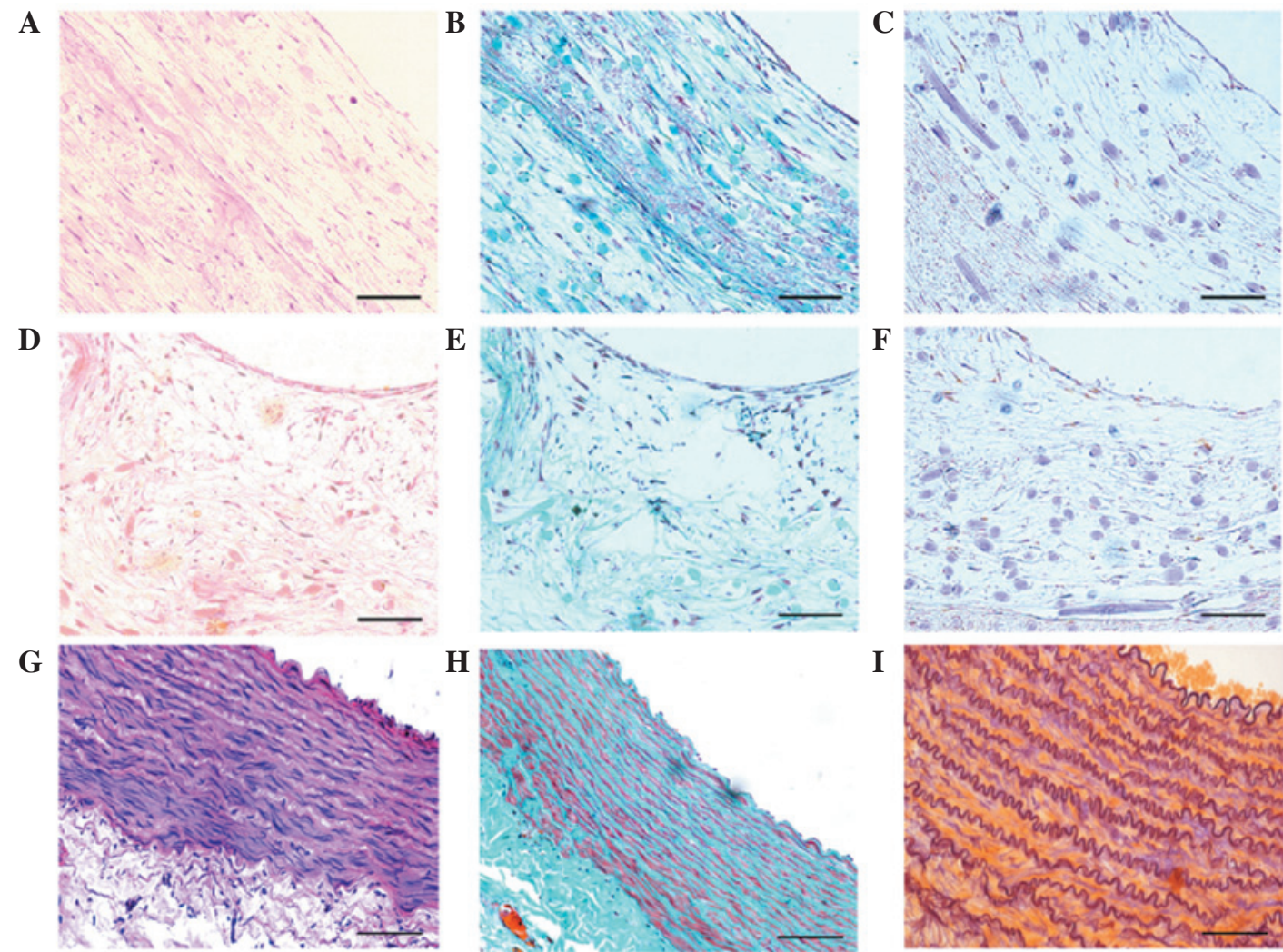

Figure 5. Histology of the engineered vessel walls after four weeks of culture (A-C) with pulsatile stimulation or (D-F) without pulsatile stimulation. (A) HE staining shows several layers of smooth muscle-like fibers with several non-degraded polyglycolic acid fibers in dynamic groups. (B and C) Masson and Gomori staining shows a few collagen and elastic fibers in the dynamic groups. (D-F) By contrast, disorganized distributed cells, randomly orientated collagenous fibers and little elastic fibers were observed in the static group. (G-I) Native canine abdominal arteries are presented for reference [stained with (G) HE, and $(\mathrm{H}, \mathrm{I})$ Masson and Gomori]. Scale bars, $50 \mu \mathrm{m}$. HE, hematoxylin and eosin.

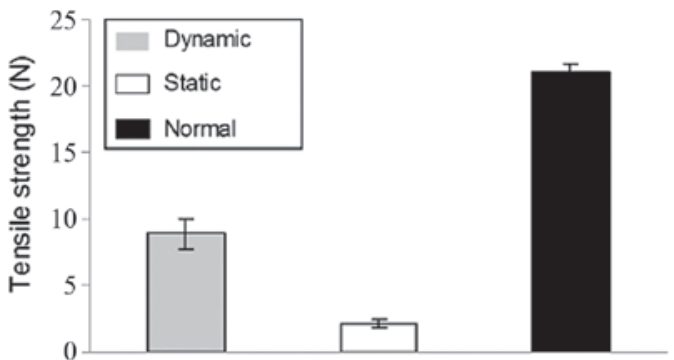

Figure 6. Tensile strength in the dynamic group was significantly higher than that in the static group $(\mathrm{P}<0.05)$ and reached nearly $41 \%$ of the tensile strength of native canine abdominal arteries (Normal) after four weeks of culture. Values are expressed as the mean \pm standard deviation of four experiments.

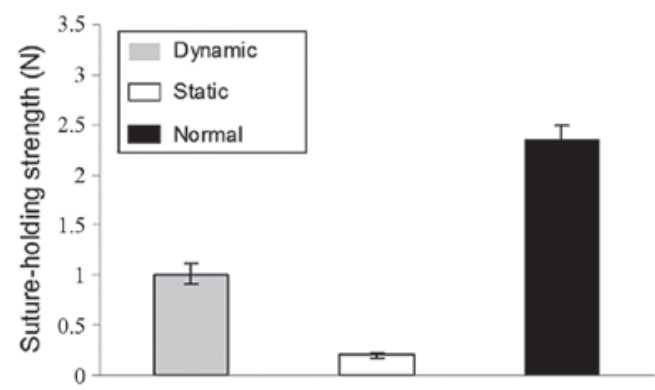

Figure 7. Suture-holding strength in the dynamic group was higher than that in the static group $(\mathrm{P}<0.05)$ and reached $\sim 42 \%$ of the suture-holding strength in normal vessels (Normal) after dynamic culture. Values are expressed as the mean \pm standard deviation of four experiments.

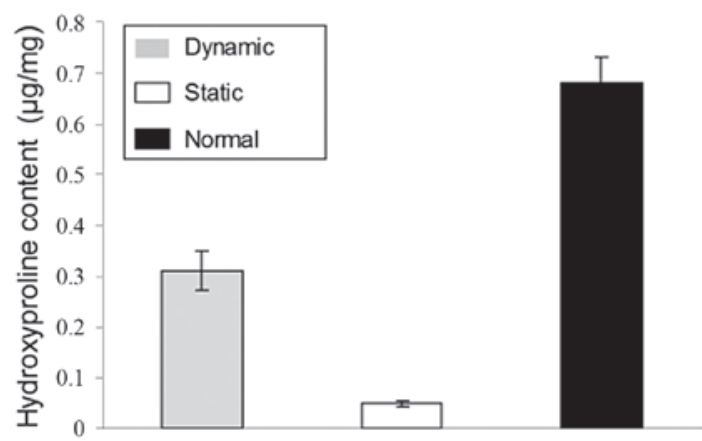

Figure 8. Quantity of hydroxyproline in the dynamic group was higher than that in the static group $(\mathrm{P}<0.05)$ and was $\sim 46 \%$ of the hydroxyproline content of the normal vessels after four weeks of dynamic culture. Values are expressed as the mean \pm standard deviation $(n=4)$.

four weeks (Fig. 3D). In the dynamic group, the constructs demonstrated a glossy and tubular structure with a round lumen of $6 \mathrm{~mm}$ in diameter (Fig. 4A). The engineered vessel in the dynamic group already exhibited excellent elasticity after four weeks of culture. The vessel wall had the capacity to repeatedly rebound to its original shape after deformation to a flat shape with tweezers. By contrast, the vessel walls in the static culture group showed a collapsed lumen and rough surface (Fig. 4B). In addition, the control vessels grown for 4 weeks in static culture failed to show any elasticity and easily collapsed upon the application of pressure. 
Histological observation. After four weeks of culture in the bioreactor, several layers of smooth muscle-like cells and a few collagenous fibres were revealed by histological examination (Fig. 5A and B). The PGA fibres did not degrade completely, and a few elastic fibres were observed at this time-point (Fig. 5C). By contrast, disorganised and randomly distributed cells, randomly organized collagenous fibres and small elastic fibres were observed in the static group (Fig. 5D-F). The above results were further confirmed by immunohistochemical staining for $\alpha$-SMA and calponin (data not shown), using a canine abdominal artery as a positive control (Fig. 5G-I).

Biomechanical testing and hydroxyproline assay. There were significant differences in the biomechanical properties between the dynamic and static groups. After being cultured for four weeks, the tensile strength (Fig. 6) and the suture-holding retention strength (Fig. 7) of the dynamic group were much higher $(\mathrm{P}<0.05)$ than those in the static group. In addition, the biomechanical properties of the dynamic group attained nearly $40 \%$ of those of the native canine abdominal arteries.

The hydroxyproline content of the dynamic group was significant higher $(\mathrm{P}<0.05)$ than that in the static group at the same time-points (Fig. 8). The hydroxyproline concentration in the dynamic group reached $\sim 40 \%$ of that in the native canine abdominal arteries.

\section{Discussion}

Numerous studies have demonstrated the potential ability of BMCs to differentiate into vascular cell types and serve as 'seed cells' in vitro (14,23-28). In such applications, BMCs may require an extended period during which they are seeded and cultured in vitro prior to being implanted in vivo. In the present study, the cultured BMCs, without any requirement for additional cytokines or transgenic technology, were used to construct an elastic large muscular vessel wall under pulsatile stimulation in a bioreactor. After culture, the newly formed tissues showed SM cell-like characteristics, including morphological indicators, the expression of SM cell-specific cytoskeletal markers ( $\alpha$-SMA and calponin) and fine biomechanical properties. These results demonstrated that BMCs are able to differentiate into SM or SM-like cells in vitro and may be utilised as a potential cell source for the tissue engineering of SMs, particularly that of large-diameter aortas.

The ideal vascular substitute should be non-thrombogenic and resistant to infections as well as possess good biocompatibility, biological characteristics, sufficient mechanical properties and compliance that match those of the native vessels, as well as be able to respond to physiological stimuli with vasomotion and remodelling (29). As to the repair of large vessels under high blood pressure, strong mechanical and haemodynamic properties are of great importance for the success of the substitute, which has been shown in the successful application of synthetic materials, including Dacron and ePTFE, even in the absence of endothelial cell sheeting (30,31). Previous studies have indicated that good mechanical properties are most important in the engineering of large blood vessels $(1,4,29)$.
To create novel and functional TEBVs in vitro, cells were seeded onto biodegradable polymer scaffolds in the present study. An optimal polymer scaffold supports biological tissue growth by providing the proper surface for cell attachment, proliferation, differentiation and the secretion of extracellular ECM proteins, as well as by directing new tissue formation. PGA is one of the most widely used polymer scaffold materials in the engineering of numerous types of tissue, including blood vessels, as it undergoes rapid degradation to soluble, safe by-products through the chemical hydrolysis of the chain ester linkages and supports cellular adhesion and collagenous matrix deposition $(32,33)$. However, rapidly degrading PGA may substantially damage the tissue mechanics. This effect was apparent in the control group of the present study.

To overcome this limitation, several vessel bioreactors have been developed and utilised for vascular tissue engineering with the aim of mimicking a suitable physiological environment for the arterial vessel wall (6,34-37). The blood's mechanical force is an important physiological component of the environment experienced by cells: It promotes the circumferential orientation of the cells as well as the deposition of the extracellular matrix and likely contributes to the survival of the implanted substitute. Several studies reported on the effects of the cyclic strain observed in smooth muscle cells, whose responses demonstrated an increased production of growth factors, enhanced synthesis or proliferation of the matrix and an increased content of contractile protein (38-40). Kim and Mooney found more elastin and contractile SMCs in the constructs cultured under a cyclic strain than in the controls (no strain) (41). Stegemann et al (42) showed that four days of exposure to periodic mechanical strain increased aortic SMC proliferation in rats. Hamilton et al (43) demonstrated that cyclic strain can be used to stimulate the expression of SMC-specific genes in primary BMCs. Similarly, the results of the present study demonstrated that the engineered vessel walls exhibited improved biomechanical properties following pulsatile stimulation with cyclic mechanical training in the bioreactor compared with those of vessels cultured under static conditions. Furthermore, the present study confirmed that vessels grown in dynamic culture exhibited good orientation of smooth muscle layers and even organization of the collagenous fibres according to histological observation.

In elastic and muscular arteries, elastin is a critical structural and regulatory matrix protein that has an important and dominant role in conferring elasticity to the vessel wall (44). Elastin also regulates vascular smooth muscle cell activity and phenotype $(40,45)$. Several scaffolds have been studied to promote elastin biosynthesis. Long and Tranquillo (46) demonstrated that more elastin was secreted by SMCs on fibrin gels than on collagen gels. According to a study by Ramamurthi and Vesely (47), a scaffold composed of hyaluron gels cross-linked with divinyl sulfone had the ability to increase the amount of elastin secreted by the SMCs. Thomas and Nair (48) showed promising results according to which the SMCs exhibited increased elastin production on a co-polymer of natural biodegradable gelatin with vinyl acetate (GeVAc) after mechanical stimulation. These studies indicated that a scaffold can have a significant impact on the synthesis of elastin. Therefore, further studies focusing on modifying PGA 
fibres with fibrin or cross-linking it with other promising materials may be beneficial for engineering improved vessel walls with a higher elastin content.

In the present study, Masson staining of artery sections for the identification of SMCs revealed red staining of native arteries, while the staining was faint in the construct cells, indicating that the nutrient supply in the engineered vessel was poor. As the vessel grew while being wrapped around a silicone tube, the nutrients in the culture media are required to pass through the thick vessel wall to reach the inner part. This implies that the thickness of the engineered vessel wall was limited in the culture system of the present study. Further improvements in the culture system are required in future studies.

Several previous studies have demonstrated that the mechanical stimulation of cells has significant effects on the cell phenotype, ECM deposition and mechanical properties of the graft (48-52). An optimal culture strategy should facilitate the efficient regeneration of vascular tissue. The stimulation of BMCs with a $10 \%$ strain for 7 days was reported to obviously upregulate the expression of $\alpha$-SMA and h1-calponin (48). In addition, Oluwole et al (53) demonstrated that the cyclic deformation of a 350- and 2,500- $\mu$ strain applied at $1 \mathrm{~Hz}$ to 10 -day-old bone marrow cultures resulted in elevated levels of alkaline phosphatase and prostacyclins. In the present study, a 5\% radial distension of the vessels was achieved using a rate of 75 beats/min, which is similar to the circulation conditions in native adult vessels in vivo. The mechanical properties of the vessel walls engineered under the dynamic culture were significantly higher than those cultured under the static conditions but did not attain the same parameters as the native arteries. Therefore, it is necessary to plan an advanced control strategy and optimise the regeneration process of the vascular tissue inside the vessel bioreactor. Specifically, future studies should address the pulse rate, deformation rate, pulsatile pressure and culture duration.

In conclusion, an elastic large vessel wall $(6 \mathrm{~mm}$ in diameter) was constructed in the present study using a PGA seeded with BMCs under pulsatile flow conditions in a vessel bioreactor. The dynamically cultured constructs exhibited a superior tissue constitution according to histological examination, as well as enhanced mechanical properties compared with the constructs that were grown in stationary culture. Further studies will focus on seeding endothelial cells on the surface of the lumen to engineer composite vascular conduits and to repair canine aortic defects.

\section{Acknowledgements}

The present study was supported by the National Natural Science Foundation of China (grant no. 81000842). The authors would like to thank Professor Hong Li (Department of Life Information and Instrument Engineering, Hangzhou Electronic Science and Technology University, Hangzhou, China), and Demin Ying, Lijuan Zong and Bing Zhong (laboratory technicians of the Department of Plastic and Reconstructive Surgery, Shanghai 9th People's Hospital, School of Medicine, Shanghai Jiaotong University, Shanghai, China) for their technical support.

\section{References}

1. Naito Y, Shinoka T, Duncan D, Hibino N, Solomon D, Cleary M, Rathore A, Fein C, Church S and Breuer C: Vascular tissue engineering: Towards the next generation vascular grafts. Adv Drug Deliv Rev 63: 312-323, 2011.

2. Khait L and Birla RK: Bypassing the patient: Comparison of biocompatible models for the future of vascular tissue engineering. Cell Transplant 21: 269-283, 2012.

3. Wang F, Mohammed A, Li C, Ge P, Wang L and King MW: Degradable/non-degradable polymer composites for in-situ tissue engineering small diameter vascular prosthesis application. Biomed Mater Eng 24: 2127-2133, 2014.

4. Woods I and Flanagan TC: Electrospinning of biomimetic scaffolds for tissue-engineered vascular grafts: Threading the path. Expert Rev Cardiovasc Ther 12: 815-832, 2014.

5. Weinberg CB and Bell E: A blood vessel model constructed from collagen and cultured vascular cells. Science 231: 397-400, 1986

6. Wang Y, Hu J, Jiao J, Liu Z, Zhou Z, Zhao C, Chang LJ, Chen YE, Ma PX and Yang B: Engineering vascular tissue with functional smooth muscle cells derived from human iPS cells and nanofibrous scaffolds. Biomaterials 35: 8960-8969, 2014.

7. Niklason LE, Gao J, Abbott WM, Hirschi KK, Houser S, Marini R and Langer R: Functional arteries grown in vitro. Science 284: 489-493, 1999.

8. Watanabe M, Shin'oka T, Tohyama S, Hibino N, Konuma T, Matsumura G, Kosaka Y, Ishida T, Imai Y, Yamakawa M, et al: Tissue-engineered vascular autograft: Inferior vena cava replacement in a dog model. Tissue Eng 7: 429-439, 2001.

9. Nerem RM and Seliktar D: Vascular tissue engineering. Ann Rev Biomed Eng 3: 225-243, 2001.

10. Galmiche MC, Koteliansky VE, Brière J,Hervé P and Charbord P: Stromal cells from human long-term marrow cultures are mesenchymal cells that differentiate following a vascular smooth muscle differentiation pathway. Blood 82: 66-76, 1993.

11. Kashiwakura Y, Katoh Y, Tamayose K, Konishi H, Takaya N, Yuhara S, Yamada M, Sugimoto K and Daida H: Isolation of bone marrow stromal cell-derived smooth muscle cells by a human SM22alpha promoter: In vitro differentiation of putative smooth muscle progenitor cells of bone marrow. Circulation 107: 2078-2081, 2003.

12. Shimizu K, Sugiyama S, Aikawa M, Fukumoto Y, Rabkin E, Libby P and Mitchell RN: Host bone-marrow cells are a source of donor intimal smooth-muscle-like cells in murine aortic transplant arteriopathy. Nat Med 7: 738-741, 2001.

13. Gong Z, Calkins G, Cheng EC, Krause D and Niklason LE: Influence of culture medium on smooth muscle cell differentiation from human bone marrow derived mesenchymal stem cells. Tissue Eng Part A 15: 319-330, 2009.

14. Wang N, Ren GD, Zhou Z, Xu Y, Qin T, Yu RF and Zhang TC: Cooperation of myocardin and smad 2 in inducing differentiation of mesenchymal stem cells into smooth muscle cells. IUBMB Life 64: 331-339, 2012.

15. Kusuma S, Facklam A and Gerecht S: Characterizing human pluripotent stem cell-derived vascular cells for tissue engineering applications. Stem Cells Dev 24: 451-458, 2015.

16. Prockop DJ: Marrow stromal cells as stem cells for nonhematopoietic tissues. Science 276: 71-74, 1997.

17. Pittenger MF, Mackay AM, Beck SC, Jaiswal RK, Douglas R, Mosca JD, Moorman MA, Simonetti DW, Craig S and Marshak DR: Multilineage potential of adult human mesenchymal stem cells. Science 284: 143-147, 1999.

18. Krause DS, Theise ND, Collector MI, Henegariu O, Hwang S, Gardner R, Neutzel S and Sharkis SJ: Multi-organ, multi-lineage engraftment by a single bone marrow-derived stem cell. Cell 105: 369-377, 2001.

19. Colter DC, Sekiya I and Prockop DJ: Identification of a subpopulation of rapidly self-renewing and multipotential adult stem cells in colonies of human marrow stromal cells. Proc Natl Acad Sci USA 98: 7841-7845, 2001.

20. Fujiwara H, Oda K, Saiki Y, Sakamoto N, Ohashi T, Sato M, Tabata Y and Tabayashi K: The wrapping method using biodegradable felt strips has a preventive effect on the thinning of the aortic wall: Experimental study in the canine aorta. J Vasc Surg 43: 349-356, 2006.

21. Schaner PJ, Martin ND, Tulenko TN, Shapiro IM, Tarola NA, Leichter RF, Carabasi RA and Dimuzio PJ: Decellularized vein as a potential scaffold for vascular tissue engineering. J Vasc Surg 40: 146-153, 2004. 
22. Reddy GK and Ewemeka CS: A simplified method for the analysis of hydroxy proline in biological tissues. Clin Biochem 29: 225-229, 1996

23. Cho SW, Kim IK, Lim SH, Kim DI, Kang SW, Kim SH, Kim YH, Lee EY, Choi CY and Kim BS: Smooth muscle-like tissues engineered with bone marrow stromal cells. Biomaterials 25 : 2979-2986, 2004.

24. Gong Z and Niklason LE: Small-diameter human vessel wall engineered from bone marrow-derived mesenchymal stem cells (hMSCs). FASEB J 22: 1635-1648, 2008.

25. Zhang G, Drinnan CT, Geuss LR and Suggs LJ: Vascular differentiation of bone marrow stem cells is directed by a tunable three-dimensional matrix. Acta Biomater 6: 3395-3403, 2010.

26. Sekiguchi H, Ii M, Jujo K, Yokoyama A, Hagiwara N and Asahara T: Improved culture-based isolation of differentiating endothelial progenitor cells from mouse bone marrow mononuclear cells. PloS One 6: e28639, 2011

27. van den AkkerNM, KolkFF, Jeukens F, Verbruggen S, Gagliardi M, Dullens S, Heschel I, Post MJ, Molin DG and Waltenberger J: Vascular potency of Sus scrofa bone marrow-derived mesenchymal stem cells: A progenitor source of medial but not endothelial cells. Tissue Eng Part A 18: 828-839, 2012.

28. Roh JD, Brennan MP, Lopez-Soler RI, Fong PM, Goyal A, Dardik A and Breuer CK: Construction of an autologous tissue-engineered venous conduit from bone marrow-derived vascular cells: Optimization of cell harvest and seeding techniques. J Pediatr Surg 42: 198-202, 2007.

29. Xu H, Su J, Sun J and Ren T: Preparation and characterization of new nano-composite scaffolds loaded with vascular stents. Int J Mol Sci 13: 3366-3381, 2012.

30. Friedman SG, Lazzaro RS, Spier LN, Moccio C and Tortolani AJ: A prospective randomized comparison of Dacron and polytetrafluoroethylene aortic bifurcation grafts. Surgery 117: 7-10, 1995.

31. Peck M, Gebhart D, Dusserre N, McAllister TN and L'Heureux N: The evolution of vascular tissue engineering and current state of the art. Cells Tissues Organs 195: 144-158, 2012.

32. Pankajakshan D and Agrawal DK: Scaffolds in tissue engineering of blood vessels. Can J Physiol Pharmacol 88: 855-873, 2010.

33. Gui L, Zhao L, Spencer RW, Burghouwt A, Taylor MS, Shalaby SW and Niklason LE: Development of novel biodegradable polymer scaffolds for vascular tissue engineering. Tissue Eng Part A 17: 1191-1200, 2011.

34. Engbers-Buijtenhuijs P, Buttafoco L, Poot AA, Dijkstra PJ, de Vos RA, Sterk LM, Geelkerken RH, Vermes I and Feijen J: Biological characterization of vascular grafts cultured in a bioreactor. Biomaterials 27: 2390-2397, 2006.

35. Arrigoni C, Chittò A, Mantero S and Remuzzi A: Rotating versus perfusion bioreactor for the culture of engineered vascular constructs based on hyaluronic acid. Biotechnol Bioeng 100: 988-997, 2008.

36. Song Y, Wennink JW, Kamphuis MM, Sterk LM, Vermes I, Poot AA, Feijen J and Grijpma DW: Dynamic culturing of smooth muscle cells in tubular poly (trimethylene carbonate) scaffolds for vascular tissue engineering. Tissue Eng Part A 17: $381-387,2011$
37. Couet F and Mantovani D: A new bioreactor adapts to materials state and builds a growth model for vascular tissue engineering. Artif Organs 36: 438-445, 2012.

38. Seliktar D, Nerem RM and Galis ZS: The role of matrix metalloproteinase-2 in the remodeling of cell-seeded vascular constructs subjected to cyclic strain. Ann Biomed Eng 29: 923-934, 2001

39. Stegemann JP and Nerem RM: Phenotype modulation in vascular tissue engineering using biochemical and mechanical stimulation. Ann Biomed Eng 31: 391-402, 2003.

40. Nikolovski J, Kim BS and Mooney DJ: Cyclic strain inhibits switching of smooth muscle cells to an osteoblast-like phenotype. FASEB J 17: 455-457, 2003.

41. Kim BS and Mooney DJ: Scaffolds for engineering smooth muscle under cyclic mechanical strain conditions. J Biomech Eng 122: 210-215, 2000.

42. Stegemann JP, Hong H and Nerem RM: Mechanical, biochemical and extracellular matrix effects on vascular smooth muscle cell phenotype. J Appl Physiol (1985) 98: 2321-2327, 2005.

43. Hamilton DW, Maul TM and Vorp DA: Characterization of the response of bone marrow-derived progenitor cells to cyclic strain: Implications for vascular tissue-engineering applications. Tissue Eng 10: 361-369, 2004.

44. Patel A, Fine B, Sandig M and Mequanint K: Elastin biosynthesis: The missing link in tissue-engineered blood vessels. Cardiovasc Res 71: 40-49, 2006

45. Opitz F, Schenke-Layland K, Cohnert TU, Starcher B, Halbhuber KJ, Martin DP and Stock UA: Tissue engineering of aortic tissue: Dire consequence of suboptimal elastic fiber synthesis in vivo. Cardiovasc Res 63: 719-730, 2004.

46. Long JL and Tranquillo RT: Elastic fiber production in cardiovascular tissue-equivalents. Matrix Biol 22: 339-350, 2003.

47. Ramamurthi A and Vesely I: Evaluation of the matrix-synthesis potential of crosslinked hyaluronan gels for tissue engineering of aortic heart valves. Biomaterials 26: 999-1010, 2005

48. Thomas LV and Nair PD: The effect of pulsatile loading and scaffold structure for the generation of a medial equivalent tissue engineered vascular graft. Biores Open Access 2: 227-239, 2013

49. Kanda K, Matsuda T and Oka T: Mechanical stress induced cellular orientation and phenotypic modulation of 3-D cultured smooth muscle cells. ASAIO J 39: M686-M690, 1993.

50. Chiquet M, Matthisson M, Koch M, Tannheimer $M$ and Chiquet-Ehrismann R: Regulation of extracellular matrix synthesis by mechanical stress. Biochem Cell Biol 74: 737-744, 1996.

51. Kim BS and Mooney DJ: Scaffols for engineering smooth muscle under cyclic mechanical strain conditions. J Biomech Eng 122: 210-215, 2000

52. Shi ZD and Tarbell JM: Fluid flow mechanotransduction in vascular smooth muscle cells and fibroblasts. Ann Biomed Eng 39: 1608-1619, 2011.

53. Oluwole BO, Du W, Mills I and Sumpio BE: Gene regulation by mechanical forces. Endothelium 5: 85-93, 1997. 